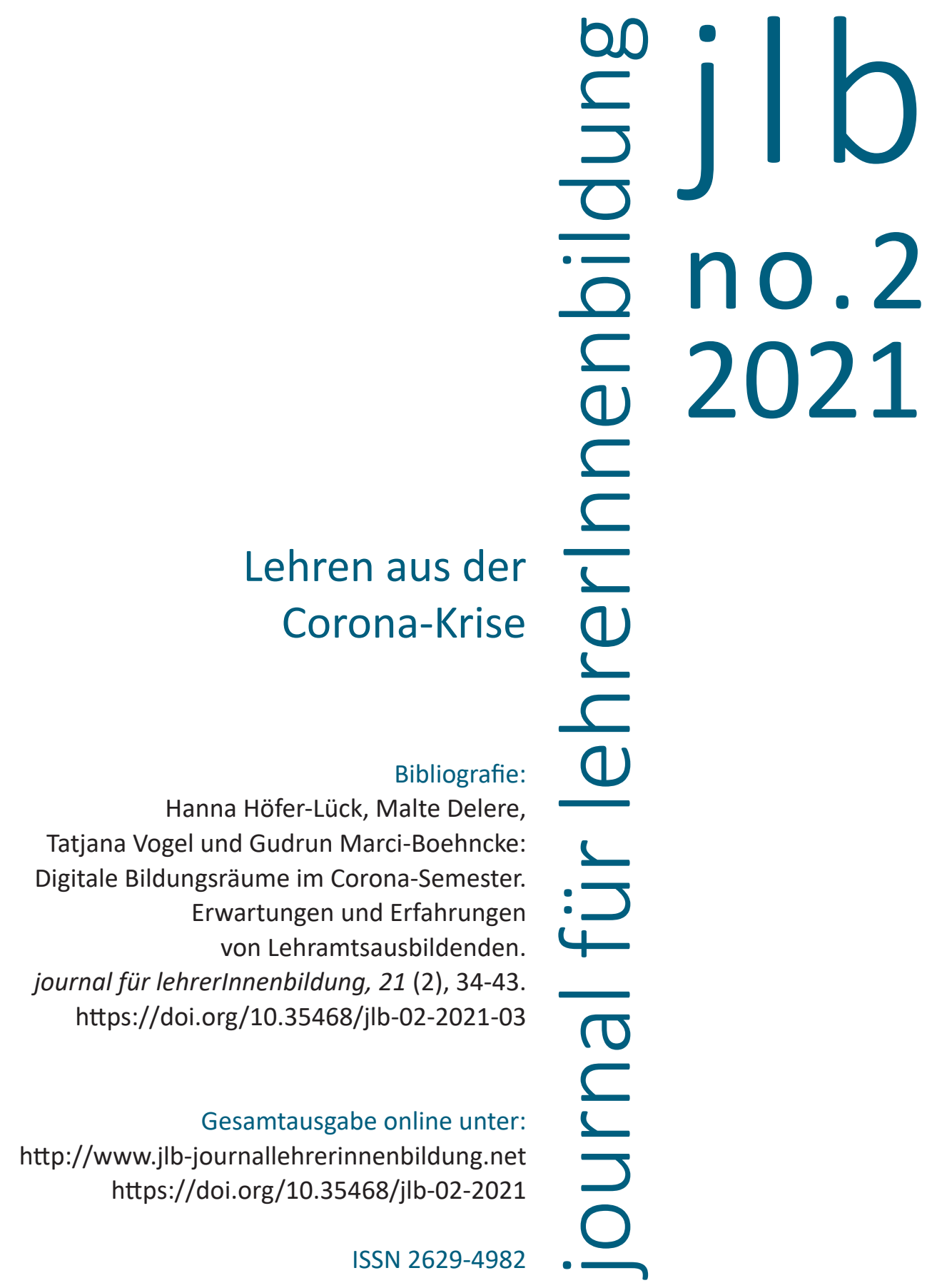


03

Hanna Höfer-Lück, Malte Delere, Tatjana Vogel und Gudrun Marci-Boehncke

\section{Digitale Bildungsräume im Corona-Semester. Erwartungen und Erfahrungen von Lehramtsausbildenden}


Im vorliegenden Artikel sollen Daten und erste Ergebnisse zur Perspektive der Lehrenden in der Lehramtsausbildung (TU Dortmund/PH Ludwigsburg) auf die Auswirkungen der Corona-Pandemie dargestellt werden. Die Studie wurde ursprünglich im Sinne eines Pre-Post-Designs mit einer Eingangs- und einer Abschlussbefragung konzipiert. Der Rücklauf des Abschlussfragebogens sowie eine geringe Anzahl von valide nachvollziehbaren Teilnehmer*innen (Überschneidung $22 \%$ ) beider Befragungen bedingen allerdings die Entscheidung, beide Erhebungen separiert wahrzunehmen und auszuwerten, um keine inadäquaten Kohärenzen zu generieren. Die Ergebnisse beider Messzeitpunkte werden hier daher jeweils in zusammengefasster Form diskutiert, um anschließend aus der Gesamtheit der Beobachtungen Erkenntnisse für die Lehramtsausbildung hervorheben zu können.

\section{Theoretische Einordnung und Studiendesign}

Ausgehend von der Prämisse einer mediatisierten Gesellschaft (Krotz, 2017), deren Eigenheiten sich im Kontext der stetig wechselnden Lebenswelt und Lehr-Lernbedingungen, auch jene während der CoronaPandemie, ändern, steht die Nutzung digitaler Medien im Zentrum der Untersuchung. Mit der medialen Veränderung geht stets auch eine des Raumes einher (Harrison, 2018), der für die Lehre genutzt wird (Edinger \& Reimer, 2015). Dieser steht zudem in Relation zur spezifischen Form des zugrundeliegenden pädagogischen Konzepts, die jeweils spezifische Lernenden-Lehrenden-Verhältnisse mit sich bringt (Anderson \& Dron, 2011). Es konnte vermutet werden, dass die Corona-Pandemie angesichts der Notwendigkeit digital zu arbeiten bei allen Lehrenden eine Beschäftigung mit digitalen Themen und Methoden veranlassen und dementsprechend im Prozess der Digitalisierung der Hochschullehre einen sogenannten Tipping Point darstellen würde (Vladova \& Renz, 2020). Die Erwartung eines Katalysatoreffekts der Corona-Pandemie für Digitalisierungsprozesse, wird gesellschaftlich (u. a. Petersen \& Bluth, 2020; Wolff, 2020) sowie unter Studierenden (Höfer-Lück, Delere \& Vogel, 2020) breit rezipiert.

Ausgehend von der Bedeutung der medienbezogenen Einstellungen von Lehrkräften und der Schwierigkeit der Erhebung solcher Aspekte in standardisierten Verfahren, sollte die Befragung die Möglichkeit bieten, eher affektiv geprägte Befürchtungen und Erwartungen frei 
auszudrücken. Mithilfe von Likert-Skalen wurden außerdem das Mediennutzungsverhalten, der dem Einsatz digitaler Medien in der Lehre zugeschriebene Stellenwert, die didaktischen Potenziale digitaler Medien sowie potenzielle Auswirkungen der veränderten Lehr-LernBedingungen auf Lehrende und Studierende erhoben.

Der halbstandardisierte digitale Fragebogen wurde zum Ende des Semesters über verschiedene E-Mail-Verteiler an die Lehrenden der Lehramtsausbildung verteilt. Zur Auswertung wurden neben der einfachen deskriptiven Auswertung der quantitativen Daten, für die aufgrund der geringen Teilnehmendenzahl kaum Anspruch auf Repräsentativität erhoben werden kann, vor allem die Daten der offenen Antworten inhaltsanalytisch mit MAXQDA analysiert. Dabei wurde der ursprünglichen Forschungsidee eines Pre-Post-Designs insofern Rechnung getragen, dass in die Analyse der Daten des zweiten Messzeitpunktes die aus der Auswertung des Pretests gewonnenen Kategorien einbezogen wurden (zu den Ergebnissen des ersten Messzeitpunkts vgl. Delere, Höfer-Lück, Marci-Boehncke \& Vogel, 2021). Darüber hinaus wurden induktiv neue Kategorien aus dem Material gewonnen.

\section{Körper und Blick im digitalen Lernraum}

Die Erwartungen, die die Lehrenden zum ersten Erhebungszeitpunkt formulierten, können den drei Bereichen Qualität der Lehre, Arbeitsbelastung und Auslösung nachhaltiger Veränderungsprozesse zugeordnet werden. Generell ließ die Analyse der offenen Antworten eine gewisse Skepsis der Lehrenden erkennen. Die Erwartung des Qualitätsverlusts wird einerseits auf die nicht ausreichende technische Ausstattung aller Akteur*innen und überlastete technische Systeme zurückgeführt. Andererseits zeigen die Lehrenden die Angst, die Lehrqualität aufgrund fehlender "charismatischer face-to-face Kontakte" (T1_13) nicht aufrechterhalten zu können, und dass durch den Einsatz digitaler Medien die "akademische Auseinandersetzung" (T1_30) leiden wird. Weiterhin wird befürchtet, dass die Betreuung der Studierenden nicht ausreichend gewährleistet werden kann (z. B. T1_111). Diese Vorstellung eines Lehr-Lernsettings ist dezidiert eine analoge. Es wird davon ausgegangen, dass die mediale Vermittlung durch in der Kommunikationssituation dazwischen geschaltete Geräte wie eine Barriere oder ein Filter fungiert, die den ,echten' Kommunikati- 
onsraum konterkarieren. Würde Raum, wie beispielsweise von Schroer (2003, S. 234) als ein „Erzeugtes“ verstanden, das „durch soziale Praktiken“ entsteht, dann wäre diese Befürchtung hinfällig (ebd.). Die Universitätslehrenden jedoch haben Sorge, Lehrinhalte aufgrund fehlender face-to-face Kontaktmöglichkeiten nicht vermitteln zu können. Sie befürchten negative Auswirkungen einer entstehenden sozialen Distanz zu den Studierenden, die hier gleichgesetzt wird mit einer physischen Distanz der Körper, sie erwarten Kontrollverlust sowie mangelnden Diskurs. Es zeigt sich in diesen Aussagen das Primat des Körpers als Medium. Eine „leibliche[] Ko-Präsenz" wie Fischer-Lichte (2004, S. 58, in Anlehnung an Herrmann 1981 [1920], S. 19f.; auch Edinger \& Reimer (2015) verweisen unter Rückgriff auf MerleauPonty (1974) auf das Verhältnis von Leiblichkeit und Apperzeption im „materielle[n] Raum“, S. 206, H. i. O.) für theatrale Prozesse, genauer die „Aufführung" (Fischer-Lichte, 2004, S. 58) für wesentlich erachtet, wird auch von den Lehrenden als zentral angesehen. Dem digitalen Raum wird in Relation zum analogen ein Manko zugeschrieben; hier sind die Sinneswahrnehmungen des anderen Körpers begrenzt. Physische und soziale Distanz werden damit im Vorfeld des Semesters gleichgesetzt. Es zeigt sich ein analog geprägtes Raumverständnis, das potenziell habituell verankert ist.

Gerade theaterwissenschaftliche Diskurse können im Kontext eines Verständnisses aktueller Lehr-Praktiken, in denen sich das Verhältnis von Körperpräsenz, Raum und Sehen verändert hat, fruchtbar sein. Haß (2005) arbeitet in ihrer Studie Das Drama des Sehens. Auge, Blick und Bühnenform für die Theaterbühne kritisch heraus, dass jene oftmals als identisch mit einem „Raum" oder „Bild“ angesehen wird (ebd., S. 16). Eine ähnliche Gleichsetzung sehen wir hinsichtlich der digitalen Lehr- und Lernräume zumindest von Videotools.

Denn der digitale Lernraum sollte ebenfalls als eine „intelligible Form“ angesehen werden „in der sich ein spezifisches Wissen über den Zusammenhang von Wahrnehmen und Darstellen ausdrückt und herstellt" (ebd., S. 17); bzw. als ein Raum, in dem es zwar einerseits um das „Wahrnehmen" (ebd.) geht, aber im Kontext didaktischer Prozesse eben auch um das Diskutieren, Produzieren und die demokratische Partizipation aller Akteur*innen an der Raumkonstruktion. Es geht also immer wieder darum, was „von verschiedenen sozialen Akteuren zu Räumen verknüpft und synthetisiert wird“ (Reißmann, 2013, S. 98; hierzu auch: Löw \& Sturm, 2005) - das heißt, der digitale Lernraum ist 
„fluid“, temporär (Harrison, 2018, o. S.; hierzu auch Edinger \& Reimer, 2015) und damit weniger steuerbar in seiner Existenz. Es geht wie von $\mathrm{Haß}$ für theatrale Prozesse herausgearbeitet, auch hier um das "Sehen“, um die "Perspektive“ (z. B. Haß, 2005, S. 17).

Trotz dieser Befürchtungen finden sich allerdings auch positive Erwartungen in den offenen Antworten, die sich unter anderem auf gegenseitige Rücksichtnahme in der Ausnahmesituation und die Aussicht auf einen digitalen Kompetenzzuwachs bei allen beteiligten Akteur*innen beziehen. Daran anknüpfend sehen die Lehrenden in der Corona-Krise die Chance, dass sich (Hoch-)Schulsysteme nachhaltig verändern und folgen damit der Wahrnehmung des oben beschriebenen Katalysatoreffektes.

\section{Analoge und digitale hybride Lernräume}

Die Antworten des zweiten Messzeitpunktes am Semesterende stellen oftmals die Erwartungen von Hybridität als Merkmal zukünftiger LehrLern-Formate in den Fokus. Diese Hybridität ist modelliert als Dichotomie zwischen analogen und digitalen Momenten, wobei, wie oben dargestellt, auch die digitale Lehre selbst analog gedacht wird und entsprechend geprägt ist. Das zeigt sich insbesondere im Raumverständnis, das sich aus den Daten des ersten Messzeitpunktes ergibt. Insgesamt lassen sich die drei Schwerpunkte Qualität der Lehre, zukünftige Lehre und Selbstwirksamkeit in den Aussagen der Lehrenden erkennen. Die Antworten auf die offenen Fragen des zweiten Fragebogens müssen allerdings als deutlich heterogen angesehen werden. Während einige Teilnehmende sehr knapp antworten, nutzen andere die Möglichkeit für sehr ausführliche und differenzierte Aussagen über ihre Erfahrungen und Erwartungen an die zukünftige Lehre.

Bezüglich der Qualität der Lehre lassen sich in den Aussagen zwei Argumentationslinien festmachen: Ein Teil der Lehrenden beschreibt einen Qualitätsverlust der Lehre und begründet dies mit einem nicht leistbaren erhöhten Zeitaufwand zur Vorbereitung (T2_39). Darüber hinaus wird eine fehlende Partizipation bemängelt. So spricht eine Person von „wenig unmittelbarem Austausch“ (T2_79) im digital durchgeführten Seminar und eine weitere von Problemen, Studierende zur Mitarbeit zu motivieren. Zusätzlich wird argumentiert, dass Studierende verunsichert seien und, dass sich im Sinne des Digital Divide (Zillien, 
2009), die Kluft zwischen Studierenden vergrößere. Ein weiterer Teil der Befragten beschreibt hingegen einen Qualitätsgewinn der Lehre, der mit einer erhöhten Partizipation der Studierenden in Chats einhergehe und positiv bewertet würde (T2_58). Zuletzt wird von einer Kompetenzsteigerung und einer neuen Offenheit für digitale Medien sowohl auf Seiten der Studierenden als auch der Lehrenden ausgegangen (T2_16).

Wie bereits angedeutet, schätzen die Lehrenden die Integration von Präsenz- und Digitallehre als zukünftig relevantes Lehrformat ein und sehen mehrheitlich "große Gewinne durch Blended-Learning und hybride Veranstaltungsmöglichkeiten" (T2_86). Offen bleibt, welche Vorstellung von Hybridität im Sinne eines Thirdspace (hierzu u. a. Edinger \& Reimer, 2015; Fraefel, 2018) dabei überhaupt vorherrscht, beziehungsweise ob von einer homogenen Vorstellung der Lehrenden diesbezüglich ausgegangen werden kann. Bereits vor der Corona-Pandemie war die grundständige Lehre hybrid angelegt: Die in Präsenz gehaltenen Lehrveranstaltungen wurden durch analoge und digitale Lerngelegenheiten ergänzt, die zum zeit- und ortsunabhängigen Lernen anhielten. Daher ist jetzt zu fragen, inwiefern durch die Corona-Pandemie wirklich eine neue Form der Hybrid-Lehre ausgelöst wird, möglicherweise begründbar durch erweiterte Kompetenzen der Studierenden und Lehrenden im Bereich der digitalen Kommunikation.

Insgesamt lassen die Aussagen des zweiten Messzeitpunkts, bis auf eine Ausnahme, die Schlussfolgerung zu, dass die Lehrenden mit dem Verlauf und den Erfahrungen des Semesters sehr zufrieden sind. Hier lässt sich vermuten, dass die Kompetenzsteigerung sowie der Erfahrungsgewinn das Selbstwirksamkeitsgefühl der Lehrenden bestärkt hat. Einige haben festgestellt, dass ihre negativen Erwartungen nicht erfüllt worden sind: „Studierende waren motivierter und lernwilliger als erwartet" (T2_19).

\section{Lehren aus der Corona-Krise?}

Das in diesem Artikel vorgestellte Forschungsprojekt sollte die digitalbezogenen Erwartungen, Befürchtungen und Erfahrungen der Lehrenden in der Lehramtsausbildung vor und nach dem Sommersemester 2020 erheben. Auch wenn die Zahl der Rückläufer im Verhältnis zur 
angesprochenen Gesamtkohorte begrenzt ist, lassen sich eindeutig in drei Bereichen Erkenntnisse bzw. eröffnete Forschungsbereiche erkennen.

Aus den Ergebnissen des ersten Erhebungszeitpunktes konnte die weitreichende Bedeutung der Wahrnehmung von digitalen Lehr- und Lernräumen erkannt werden, die sowohl im universitären als auch schulischen Bereich Bedeutung trägt und didaktische Konzeptionen determiniert. Lehrende auf allen Bildungsebenen müssen in der Ausbildung ein Selbstverständnis als aktiv Konstruierende ihres eigenen Lernraumes entwickeln und reflektieren, um im Laufe ihrer professionellen Tätigkeit adäquat auf gesellschaftliche Veränderungen reagieren zu können. Damit einher geht die Notwendigkeit, den Lernenden ihre partizipative Rolle als „Jongleurinnen und Jongleure“ von „Raumkomponenten" (Edinger \& Reimer, 2015, S. 210) zuzugestehen. Noch zu klären bleibt, inwiefern ein spezifisches Raumverständnis in habituellen Strukturen der Lehrkräfte verankert ist.

Die Ergebnisse des zweiten Erhebungspunktes zeigen anhand der dargestellten positiven Selbstwirksamkeitserfahrungen die Relevanz von aktivem digitalen Handeln in der Lehramtsausbildung auf. Darüber hinaus stellt sich die oben beschriebene Frage, inwiefern hybride Lehre von den Lehrenden als Format gedacht wird, dessen digitale Momente nicht nur als Abbilder analoger Verfahren und Lernräume konzipiert werden. Die Umsetzung konsequent hybrider Lehre, d. h. mit digitalen Elementen, die den veränderten Bedingungen und Potenzialen digitaler Lehr- und Lernformate gerecht werden, erfordert dabei von Universitätslehrenden als Multiplikator*innen für künftige Lehrkräfte und damit auch deren Unterricht, das Zulassen aktiver medialer Handlungen und veränderter digitaler Raumkonzepte.

\section{Literatur}

Anderson, T. \& Dron, J. (2011). Three Generations of Distance Education Pedagogy. International Review of Research in Open and Distance Learning, 12(3), 80-97.

Delere, M., Höfer-Lück, H., Marci-Boehncke, G. \& Vogel T. (2021). Analog vs. Digital Spaces - How University Lecturers Evaluate Possibilities for Pre-Service Teachers Education within the Corona-Pandemic. INTED21 Conference Proceedings, 34253432.

Edinger, E.-C. \& Reimer, R. (2015). Thirdspace als hybride Lernumgebung. Die Kombination materieller und virtueller Lernräume. In C. Bernhard, K. Kraus, S. SchreiberBarsch \& R. Stang (Hrsg.), Erwachsenenbildung und Raum. Theoretische Perspek- 
tiven - professionelles Handeln - Rahmung des Lernens. Theorie und Praxis der Erwachsenenbildung (S. 205-216). Bielefeld: Deutsches Institut für Erwachsenenbildung.

Fischer-Lichte, E. (2004). Ästhetik des Performativen. Frankfurt am Main: Suhrkamp.

Fraefel, U. (2018). Hybride Räume an der Schnittstelle von Hochschule und Schulfeld. In L. Pilypaitytė \& H.-S. Siller (Hrsg.), Schulpraktische Lehrerprofessionalisierung als Ort der Zusammenarbeit (S. 13-43). Wiesbaden: Springer VS.

Harrison, M. (2018). Space as a tool for analysis: Examining digital learning. International Council for Open and Distance Education. Verfügbar unter https://open praxis.org/index.php/OpenPraxis/article/view/782/429 [23.12.2020].

Haß, U. (2005). Das Drama des Sehens. Auge, Blick und Bühnenform. München: Fink. Herrmann, M. (1981 [1920]). Über die Aufgaben eines theaterwissenschaftlichen Institutes. In H. Klier (Hrsg.), Theaterwissenschaft im deutschsprachigen Raum (S. 1522). Darmstadt: Wiss. Buchgesellschaft.

Höfer-Lück, H., Delere, M. \& Vogel, T. (2020). Changing Practices and Self-Reflection? Implications of The Corona Crisis Regarding Private and Professional Digital Media Use of Pre-Service Teachers. EDULEARN20 Proceedings, 7319-7327.

Krotz, F. (2017). Sozialisation in mediatisierten Welten. In D. Hoffmann, F. Krotz \& W. Reißmann (Hrsg.), Medien und Mediensozialisation. Prozesse - Räume - Praktiken (S. 21-40). Wiesbaden: VS Verlag für Sozialwissenschaften.

Löw, M. \& Sturm, G. (2005). Raumsoziologie. In F. Kessl, C. Reutlinger, S. Maurer \& O. Frey (Hrsg.), Handbuch Sozialraum (S. 31-48). Wiesbaden: Springer VS.

Merleau-Ponty, M. (1974). Phänomenologie der Wahrnehmung. Berlin: De Gruyter.

Petersen, T. \& Bluth, C. (2020). Megatrend-Report \#02: Die Corona-Transformation. Wie die Pandemie die Globalisierung bremst und die Digitalisierung beschleunigt. Verfügbar unter https://www.bertelsmann-stiftung.de/fileadmin/files/user_ upload/MT_MegatrendReport2_Web_2020_DT.pdf [23.12.2020].

Reißmann, W. (2013). Warum Netzwerkplattformen (keine) Räume sind: Ein Beitrag aus medienökologischer Perspektive. In T. Junge (Hrsg.), Soziale Netzwerke im Diskurs. Verfügbar unter https://ub-deposit.fernuni-hagen.de/receive/mir_ mods_00000265 [28.12.2020].

Schroer, M. (2003). Raumgrenzen in Bewegung. Zur Interpenetration realer und virtueller Räume. In C. Funken \& M. Löw (Hrsg.), Raum - Zeit - Medialität (S. 217236). Wiesbaden: VS Verlag für Sozialwissenschaften.

Vladova, G. \& Renz, A. (2020). Die Coronakrise als Katalysator für die Digitalisierung von Bildungsprozessen. Verfügbar unter https://www.weizenbaum-institut.de/ index.php?id=107\&tx_news_pi1\%5bnews\%5d=229\&tx_news_pi1\%5bcontroller $\% 5 \mathrm{~d}=$ News\&tx_news_pi $\% 5$ baction $\% 5 \mathrm{~d}=$ detail $\&$ cHash $=8 \mathrm{~b} 3 \mathrm{ad} 84965 \mathrm{c} 84 \mathrm{e} 0599 \mathrm{~b}$ 0a20b295aacfd [23.12.2020].

Wolff, S. (2020). Corona als Katalysator. Verfügbar unter https://www.tagesschau.de/ wirtschaft/boerse/hr-boerse-story-24325.html [23.12.2020].

Zillien, N. (2009). Digitale Ungleichheit: neue Technologien und alte Ungleichheiten in der Informations- und Wissensgesellschaft (2. Aufl.). Wiesbaden: Springer VS. 


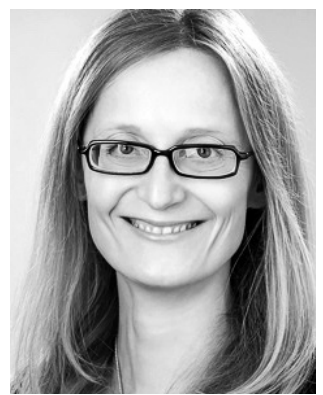

Hanna Höfer-Lück, M.A., AkadR. a. Z.

an der Forschungsstelle Jugend-Medien-Bildung, TU Dortmund.

Arbeitsschwerpunkte:

Medien-, Literatur- und Theaterdidaktik, Theater und Literatur des 20./21. Jahrhunderts, Interdisziplinarität, Ohnmacht

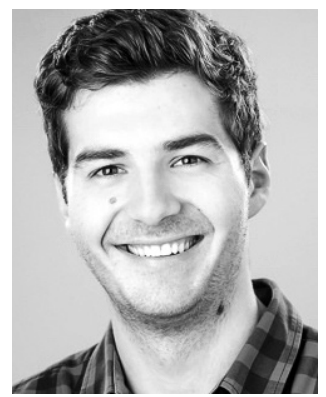

hanna.hoefer-lueck@tu-dortmund.de

Malte Delere, M.Ed., wiss. Mitarbeiter an der Forschungsstelle Jugend-Medien-Bildung, TU Dortmund.

Arbeitsschwerpunkte:

videobasierte Lehramtsausbildung,

digitale Partizipation in mediatisierten Gesellschaften

malte.delere@tu-dortmund.de 
Tatjana Vogel, M.Ed., wiss. Mitarbeiterin an der Forschungsstelle Jugend-Medien-Bildung, TU Dortmund. Arbeitsschwerpunkte: Digital Literacy, Literaturdidaktik, Medienforschung

tatjana.vogel@tu-dortmund.de

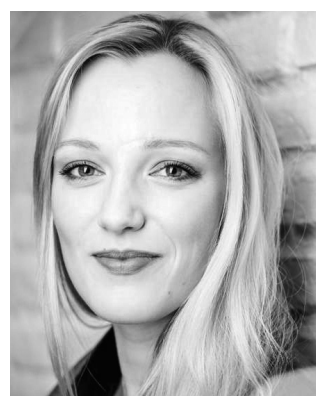

Gudrun Marci-Boehncke, Prof. Dr.phil., Leiterin der Forschungsstelle Jugend-Medien-Bildung, TU Dortmund. Arbeitsschwerpunkt: Digitale Lese- und Medien(bildungs-)forschung entlang der Bildungskette, Inklusion und Diversität

gudrun.marci@tu-dortmund.de

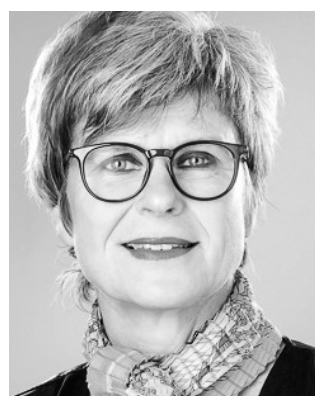

